\title{
Dexmedetomidine Directly Increases Tau Phosphorylation
}

\author{
Chunxia Huang ${ }^{\mathrm{a}, \mathrm{b}}$, Yuen-Shan $\mathrm{Ho}^{\mathrm{b}, \mathrm{e}}$, Olivia Tsz-Wa Nga, , Michael G. Irwin ${ }^{\mathrm{a}, \mathrm{c}}$, \\ Raymond Chuen-Chung Chang ${ }^{\mathrm{b}, \mathrm{c}, \mathrm{d}, *}$ and Gordon Tin-Chun Wong ${ }^{\mathrm{a}, \mathrm{c}, *}$ \\ ${ }^{a}$ Department of Anaesthesiology, LKS Faculty of Medicine, The University of Hong Kong, Pokfulam, \\ Hong Kong SAR, China \\ ${ }^{\mathrm{b}}$ Laboratory of Neurodegenerative Diseases, Department of Anatomy, LKS Faculty of Medicine, \\ The University of Hong Kong, Pokfulam, Hong Kong SAR, China \\ ${ }^{\mathrm{c}}$ Research Centre of Heart, Brain, Hormone and Healthy Aging, LKS Faculty of Medicine, \\ The University of Hong Kong, Pokfulam, Hong Kong SAR, China \\ ${ }^{\mathrm{d}}$ State Key Laboratory of Brain and Cognitive Sciences, The University of Hong Kong, Hong Kong SAR, China \\ ${ }^{\mathrm{e}}$ School of Nursing, Faculty of Health and Social Sciences, The Hong Kong Polytechnic University, \\ Hung Hom, Kowloon, Hong Kong SAR, China
}

Handling Associate Editor: Fei Liu

Accepted 25 September 2014

\begin{abstract}
Exposure to anesthetic agents has been linked to abnormal tau protein phosphorylation, an antecedent to the development of neurofibrillary tangles. This study evaluates the direct and indirect effects of dexmedetomidine. Primary culture of cortical neurons established from Sprague-Dawley (SD) rat embryos were exposed to dexmedetomidine for 1 or 6 hours, and the degree of tau phosphorylation at the AT8, AT180, and S396 sites was assessed by western blot analysis. To assess and compare their relative in vivo effects, the same agent was administered intravenously to 8 to 10 week old male SD rats and titrated to the loss of the righting reflex for 2 hours. After 1 hour of recovery, the rats were sacrificed and samples taken from the cortex and hippocampus were subjected to western blot and immunohistochemical analysis. The in vitro studies reviewed significant hyperphosphorylation only at the S396 site, and these changes have largely disappeared at 6 hours. With temperature maintenance, dexmedetomidine induced significant changes in hyperphosphorylation at the AT8 site in the cortex and hippocampus and at the AT180 in the hippocampus. The direct effect of anesthetic agents on fully differentiated cortical neurons is epitope-specific and short-lived. The in vivo effects are comparatively more complicated and depend not only on the phosphorylation site but the regions of the brain examined. These findings suggest that dexmedetomidine increases tau phosphorylation both in vitro and in vivo under normothermic conditions, and further studies are warranted to determine the long-term impact of this anesthetic on the tau pathology and even cognitive function.
\end{abstract}

Keywords: Anesthetics, dexmedetomidine, hypothermia, protein kinases, tau phosphorylation

\footnotetext{
${ }^{*}$ Correspondence to: Dr. Gordon T.C. Wong, MD, Department of Anaesthesiology, The University of Hong Kong, Room K424, Queen Mary Hospital, Pokfulam, Hong Kong. Tel.: +852 22553303; Fax: +852 2255 1654; E-mail: gordon@hku.hk. or Dr. Raymond Chuen-Chung Chang, Rm. L1-49, Laboratory Block, Department of Anatomy, Faculty of Medicine Building, 21 Sassoon Road, Pokfulam, Hong Kong. Tel.: +852 3917 9127; Fax: +852 2817-0857; E-mail: rccchang@hku.hk.
}

\section{INTRODUCTION}

Tau proteins are essential to the normal functions of microtubules and contribute to axonal integrity of the normal mature neuron [1]. Abnormal tau phosphorylation leads to its self-aggregation into paired helical filaments, the major component of neurofibrillary tangles, which is a prominent and consistent 
histological feature found in brains of patients afflicted with Alzheimer's disease (AD) [1].

Anesthesia exposure has been linked to the induction of abnormal tau hyperphosphorylation; and thus there is growing interest in the potential relationship between anesthesia and the progression of AD. Anesthesia exposure has also been implicated as a potential contributor to the development of postoperative cognitive dysfunction [2]. Anesthesia-induced tau hyperphosphorylation has been reported by a number of investigators using different agents and conditions [3-6]. Initially, these findings were attributed to anesthesia-induced hypothermia rather than a direct adverse effect of the agents themselves [6]. In contrast, very brief periods of anesthetic exposure have also been shown to induce some degrees of tau phosphorylation independent of temperature changes $[3,7,8]$.

Dexmedetomidine is a highly selective alpha-2adrenergic agonist which exhibits a wide range of effects on the mammalian brain that includes sedation, analgesia, and anxiolysis effects [9]. It has become a popular drug for sedation in the perioperative setting and intensive care unit (ICU), where it has exhibited a number of benefits over traditional sedatives, including a reduction in the number of days of delirium or coma in mechanically ventilated patients in the ICU $[9,10]$. This agent is also being used intra-operatively as an adjunct to general anesthesia, where it has displayed anesthetic and analgesic sparing effects. It is of note that dexmedetomidine is distinctive in that its clinical effects are achieved independent of actions on gamma aminobutyric acid (GABA) receptors, the putative target of many of the current sedative and anesthetic agents [11]. Dexmedetomidine has been shown to exert a number of neuroprotective effects via several mechanisms both in vivo and in vitro. It decreases catecholamine levels to improve cerebral oxygen supplies and perfusion in the ischemic penumbra as well as reducing excitotoxicity [12]. Stimulation of astrocytic $\alpha-2$ adrenergic receptors raises astrocytic calcium concentrations, which in turn stimulates glutaminase enzyme activity and the ability of astrocytes to dispose of glutamine by oxidative metabolism [13]. Dexmedetomidine increases the concentration of BCL-2 in the mitochondrial membrane during ischemia thereby decreasing mitochondrial membrane permeability and reducing the release of proapoptotic protease activity such as cytochrome $\mathrm{C}$ or the apoptosis-inducing factor from the mitochondria into the cytosol [14]. Inhibition of isoflurane induced capsase-3 expression in hippocampal slice cultures suggests that dexmedetomidine may be an important adjunct to prevent isoflurane-induced neurotoxicity in the growing brain [15].

In light of these putative neurologic benefits against ischemic insults, it is, therefore, of interest to delineate whether dexmedetomidine has any effects on tau phosphorylation as seen with GABA agonists. Hence, the aim of this study was to examine the direct impact of dexmedetomidine on tau phosphorylation under normothermic conditions. We found that dexmedetomidine leads to tau hyperphosphorylation under normothermic conditions both in rat cortical neuronal cells and rats.

\section{MATERIALS AND METHODS}

\section{Primary culture of cortical neurons and drug treatment}

Experimental protocols were approved by our local institutional animal ethics committee and animals were treated in accordance with $\mathrm{NIH}$ guidelines. Primary culture of cortical neurons was prepared from embryonic day $18 \pm 0.5$ Sprague-Dawley (SD) rat embryos according to our previously published method [16]. Briefly, cortices were dissected and neurons were seeded onto 6-well plates pre-coated with poly-L-lysine $(25 \mu \mathrm{g} / \mathrm{ml})$ at a density of $4.0 \times 10^{5}$ cells/well and mixed with a combination of $1: 1$ SMEM medium (MEM medium added with $0.3 \%$ glucose, $1 \%$ glutamine, $5 \%$ fetal bovine serum, penicillin $(50 \mathrm{U} / \mathrm{ml})$, and streptomycin $(50 \mu \mathrm{g} / \mathrm{ml})$ and $0.41 \%$ hormone cocktail and $\beta$-mercaptoethanol) and NB medium (Neurobasal ${ }^{\mathrm{TM}}$ medium supplemented with L-Glutamine $(2 \mathrm{mM})$, B-27 supplement, penicillin $(50 \mathrm{U} / \mathrm{ml})$, and streptomycin $(50 \mu \mathrm{g} / \mathrm{ml}))$. All reagents used for in vitro studies were from Gibco-BRL (Burlington, Ont., Canada). After $24 \mathrm{~h}$ of incubation, deoxyfluorouridine (final concentration $1 \mu \mathrm{M}$ ) was added to the cortical neuron culture to suppress the growth of proliferating glial cells. One third of the medium was replaced with NB medium every 2 days. Neurons were maintained at $37^{\circ} \mathrm{C}$ in a humidified atmosphere with $5 \% \mathrm{CO}_{2}$. All experiments were performed with 14-day-old culture, which is considered to contain differentiated neurons $[17,18]$.

Cultured neurons were exposed to one of the following treatments. In the control group $(\mathrm{CON})$, neurons were cultured and harvested in the medium without any additional treatment. As a positive control for tau phosphorylation, $50 \mathrm{nM}$ of okadaic acid (OA) was added to the medium and incubated for $3 \mathrm{~h}$ before harvest. Three dosages of dexmedetomidine (Dex; Pfizer, USA) were 
used: 5,10 , or $20 \mu \mathrm{M}$ of the drug were added into the medium for 1 or $6 \mathrm{~h}$. These doses were in the order of magnitude that had previously shown to be non-toxic to cultured neurons [19]. Finally, the effects of mild $\left(35^{\circ} \mathrm{C}\right)$ or profound $\left(30^{\circ} \mathrm{C}\right)$ hypothermia were assessed by incubating the cells in medium only at the respective temperature. For each treatment the level of phosphorylation at the AT8, AT180, and S396 were determined by western blot and the results were expressed as a ratio of total of tau proteins. Five determinations were used for each of the different treatment with each plate of cells representing one experiment.

\section{In vivo anesthetic exposure}

Fifty-four male SD rats aged between 8 and 10 weeks, weighing between 270-310 g were obtained from the Laboratory Animal Unit (LAU) of the LKS Faculty of Medicine in the University of Hong Kong, where the LAU has been accredited by the Association for Assessment and Accreditation of Laboratory Animal Care International (AAALAC). Effort has been made to reduce the number of animal to be used in the experiment. They were housed in a temperaturecontrolled room $\left(20-22^{\circ} \mathrm{C}\right)$ with a 12 -h light/dark cycle prior to experiment. The animals were divided into 4 groups randomly ( $n=7$ in each group). In the control group $(\mathrm{CON})$, the rats were allowed to become comatose without any treatment by exposure to high purity $\mathrm{CO}_{2}$ for less than $30 \mathrm{~s}$ according to the guideline of American Veterinary Medical Association (AVMA) for euthanasia. The head was then cut after decapitation. In the Sham group (Sham), tail veins of the rats were cannulated with a $24 \mathrm{G}$ intravenous catheter and were given a bolus of the vehicle control saline for the same induction time with anesthetic groups. In the dexmedetomidine groups, the animals subjected to dexmedetomidine treatment had either temperature maintained at $35^{\circ} \mathrm{C}$ (Dex35) using a heating pad or have no temperature control (Dex). The animals were induced with 1 to $2 \mathrm{ml} / \mathrm{kg}$ of drug solution $(20 \mu \mathrm{g} / \mathrm{ml})$ and maintained at $1.5 \mathrm{ml} / \mathrm{h}$, which was in the range of $1 \mu \mathrm{g} / \mathrm{kg} / \mathrm{min}$, and monitored by the loss of the righting reflect [20]. Drug was administered using a micro-pump (ALC-IP600LB, Shanghai Alcott Biotech, China) for $2 \mathrm{~h}$ continuously through a tail vein cannula. The rate of infusion was adjusted every $15 \mathrm{~min}$ as required. Body temperature was measured using an animal body temperature thermometer (BW-TH1101, ShangHai Biowill, China).

After $2 \mathrm{~h}$, the infusion was stopped and the rats were allowed to recover in their cages for $1 \mathrm{~h}$ before being sacrificed. The rats were then perfused with cold saline and the brains were quickly removed. Half of the brains were dissected into different regions for western blot analysis. The other half were fixed with $4 \%$ paraformaldehyde and dehydrated in ethanol and embedded in paraffin for immunohistochemical analysis.

\section{Western blot analysis}

Cultured neurons or brain tissues were harvested at the end of the experiment and were subjected to western blot analysis according to our previous methods $[16,21]$. Proteins were extracted in lysis buffer containing a cocktail of protease inhibitors purchased from Sigma-Aldrich, Inc. (St. Louis, MO, USA) according to our previous publications [17]. Total lysate was collected and they were subjected to SDS polyacrylamide gels electrophoresis and transferred to PVDF membranes (Bio-Rad Laboratories, Inc., California). The membranes were incubated with different monoclonal antibodies AT8, AT180 (Thermo Fisher Scientific Inc., USA), and S396 (Cell Signaling Technology, USA) recognize phosphoepitopes; which are specific for tau phosphorylated at Ser202 and Thr205 (AT8), Thr231 (AT180), and Ser396 (S396). Moreover, the protein level of several effectors involved in signaling mechanism were also investigated, which included $p$-ERK1/2, $p$-AKT (Ser473), $p$-JNK (T183/Y185), $p$ GSK3 $\beta$ (S9), $p$-GSK-3 $\beta$ (Y216) (all purchased from Cell Signaling Technology, USA), p-PP2A (Y307), and PP2A-C (Epiromomic, USA). After washing, the membranes were incubated with horseradish peroxidase (HRP)-conjugated secondary antibodies (DAKO, Denmark) for 1.5 to $2 \mathrm{~h}$ and subsequently developed by using the ECL or ECL-plus western blotting detection kit (Amersham, UK). The membranes were then stripped with stripping buffer $(50 \mathrm{mM}$ glycine, $2 \%$ SDS, pH 2.0) and re-probed with anti- $\beta$-actin antibody (Cell Signaling Technology, USA), GAPDH (Cell signaling Technology, USA), and then goat anti-mouse HRP secondary antibody. The intensity of the band was assessed and analyzed by gel documentation system UVP, Inc. (Upland, CA, USA).

\section{Immunohistochemical analysis}

Paraffin blocks were prepared from the brain samples and 6- $\mu \mathrm{m}$-thick coronal sections were cut. After dewaxing and rehydration, the sections were treated with $0.01 \mathrm{M}$ citrate buffer ( $\mathrm{pH}$ 6.0) with $0.1 \%$ Tween20 at $90^{\circ} \mathrm{C}$ for $15 \mathrm{~min}$ for antigen retrieval. After 
washing with phosphate-buffered saline, sections were blocked with $10 \%$ normal goat serum for $1 \mathrm{~h}$ in room temperature, and then incubated with primary antibodies in $4^{\circ} \mathrm{C}$ overnight. Then, the sections were incubated with AlexaFluor488 second antibodies for $2 \mathrm{~h}$ after washing with phosphate-buffered saline. Finally, they were co-stained with DAPI to reveal the nuclei. Sections were examined with a Zeiss Axioplan 2 microscope.

\section{Statistical analysis}

The western blot data are expressed as mean \pm standard derivation (SD) from 5 independent experiments in the cell cultures and from brain tissues of 6-7 animals for the in vivo studies. Normalized band intensities were analyzed by One Way ANOVA followed, if significant, by Tukey's test as post-hoc test using Quantity One, with $p<0.05$ being considered as statistically significant.

\section{RESULTS}

Dexmedetomidine increased tau phosphorylation at the 396 epitope under hypothermic and normothermic conditions in the rat cortical neurons

Cultured cortical neurons were treated with different concentrations of dexmedetomidine under normothermia at $37^{\circ} \mathrm{C}$. The results showed that dexmedetomidine has relatively little direct effects on tau phosphorylation in vitro compared with hypothermia and OA at the phosphoepitopes AT8 and AT180 antibodies. The immunoreactivities of AT8, AT180, and S396 were compared by using the ratio of their levels to total tau protein (panTau) after 1 and $6 \mathrm{~h}$ incubation (Fig. 1). Under the same conditions, none of the dosage of dexmedetomidine displayed any significant effects on the tau phosphorylation sites as recognized by the antibodies AT8 (S202/T205) or AT180 (T231) after $1 \mathrm{~h}$

a
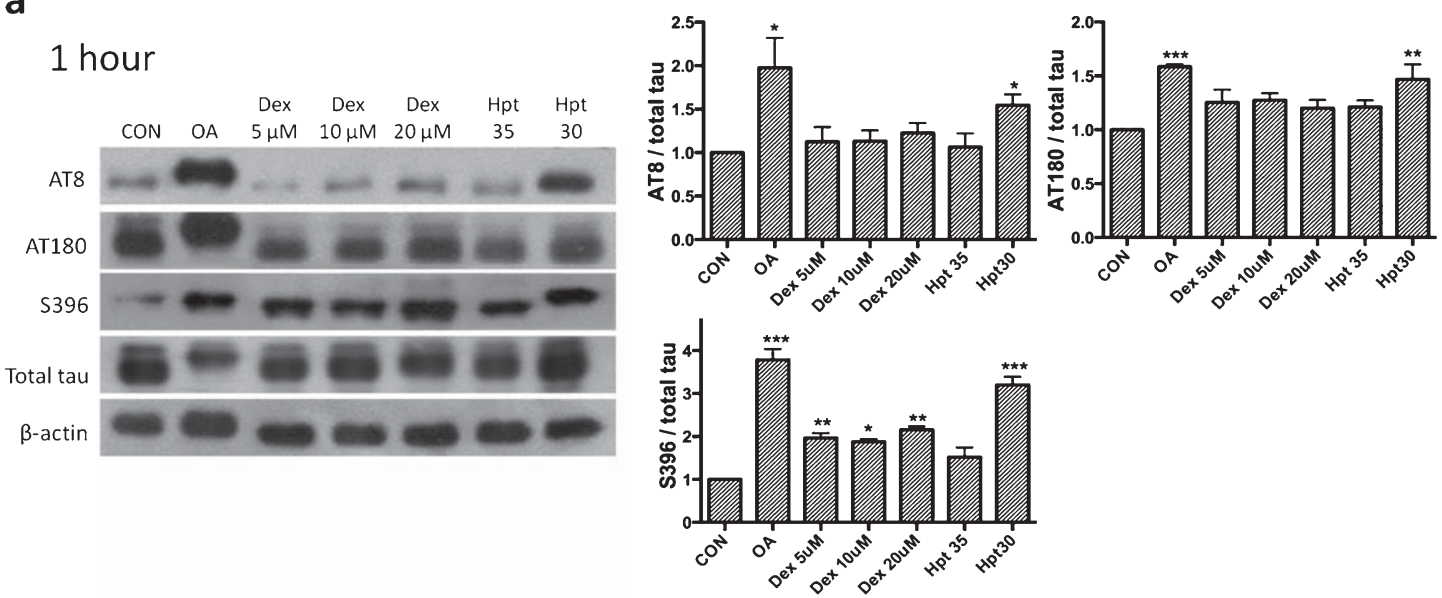

b

\section{6 hours}
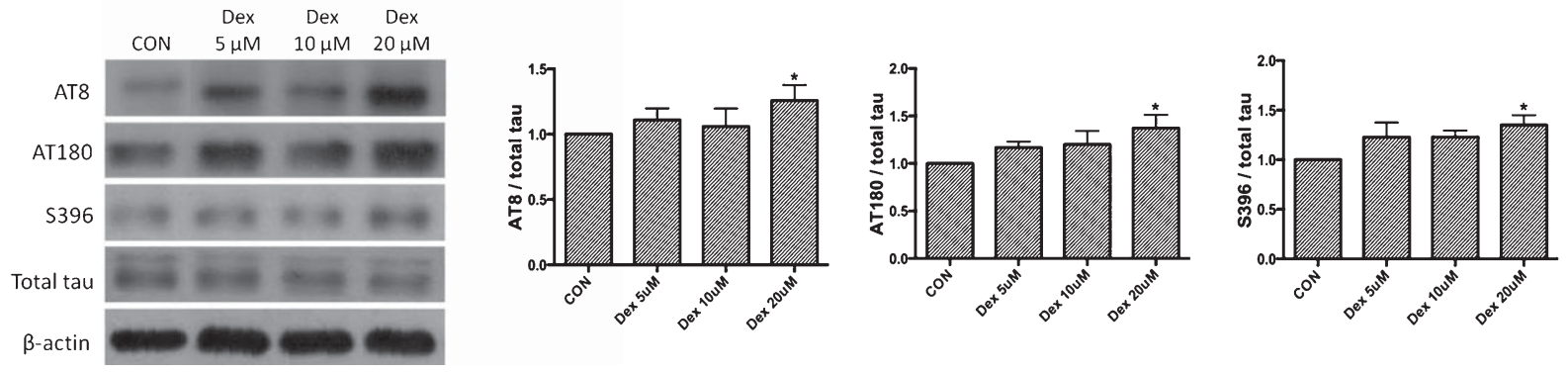

Fig. 1. Tau phosphorylation in primary culture of cortical neurons exposed to dexmedetomidine for a) one hour or b) six hours. Results are presented as ratio of the control. Tau phosphorylation was determined using antibodies directed at the AT8 (Ser202 + Thr205), AT180 (Thr231), and S396 sites. Data is expressed as mean $\pm \mathrm{SD},{ }^{*} p<0.05$ and ${ }^{* *} p<0.01$ versus CON. CON, control; OA, okadaic acid; Dex, Dexmedetomidine; Hpt, hypothermia 35 degrees; Hpt 30, hypothermia 30 degrees. 
exposure compared with the control group. In contrast, dexmedetomidine at 5 or $10 \mu \mathrm{M}$ significantly increased phosphorylation of tau at S396 after $1 \mathrm{~h}$ exposure. The increase was no longer apparent after $6 \mathrm{~h}$ exposure, except if neurons were treated with $20 \mu \mathrm{M}$ dexmedetomidine. However, significant increase in phosphorylation of tau at the AT8 and AT180 sites was observed in $20 \mu \mathrm{M}$ dexmedetomidine-treated neurons after $6 \mathrm{~h}$ exposure compared with the control group. As excessive cell death was known to occur at the $6 \mathrm{~h}$ of exposure to $\mathrm{OA}$ and hypothermia, these groups were omitted from the analysis. Our data indicated that the dexmedetomidine could induce tau phosphorylation at Ser202/Thr205, Thr231, and S396 in different periods of exposure time and higher dosages may lead to a long-term effect.

\section{Dexmedetomidine induced tau phosphorylation in the rat CA3 hippocampal but not cortical neurons under hypothermic and normothermic conditions}

Apart from in vitro experiments, the impact of dexmedetomidine on tau phosphorylation was further investigated in the intact animals. Firstly, we confirmed that dexmedetomidine could trigger hypothermiainduced phosphorylation of tau, as it has not been reported previously. In the group of rats whose body temperature was not controlled during anesthesia (Dex), administration of dexmedetomidine elicited significant hypothermia. The rat rectal temperature decreased to $33.64 \pm 0.36^{\circ} \mathrm{C}$ and $29.18 \pm 0.28^{\circ} \mathrm{C}$ after $15 \mathrm{~min}$ and $2 \mathrm{~h}$, respectively, in the hypothermia group versus $35.10 \pm 0.40^{\circ} \mathrm{C}$ at the beginning of the infusion $(p<0.001)$. Normothermia was maintained in the temperature controlled group (Dex35) with rectal temperature at $35.25 \pm 0.41^{\circ} \mathrm{C}$ based on their basic temperature level. In the Sham group, the rectal temperature was $36.1 \pm 0.68^{\circ} \mathrm{C}$, which indicated the saline infusion and the tail vein cannulation process did not result in hypothermia.

The results of immunofluorescent staining revealed that there was an increase in phosphorylation of tau at Thr231 and Ser202/Thr205 but not at S396 in CA3 hippocampus neurons after continuously infusion of dexmedetomidine for $2 \mathrm{~h}$ (Fig. 2). However, no positive immunofluorescent reactivity was observed in the cortex (data not shown). Interestingly, tau phosphorylation was increased in not only in the Dex group but also in the Dex35 group, as indicated by AT8, AT180, and DAPI staining. The TUNEL staining also revealed that there was no apoptosis or significant decrease in cell numbers in these investigated regions (data not shown).
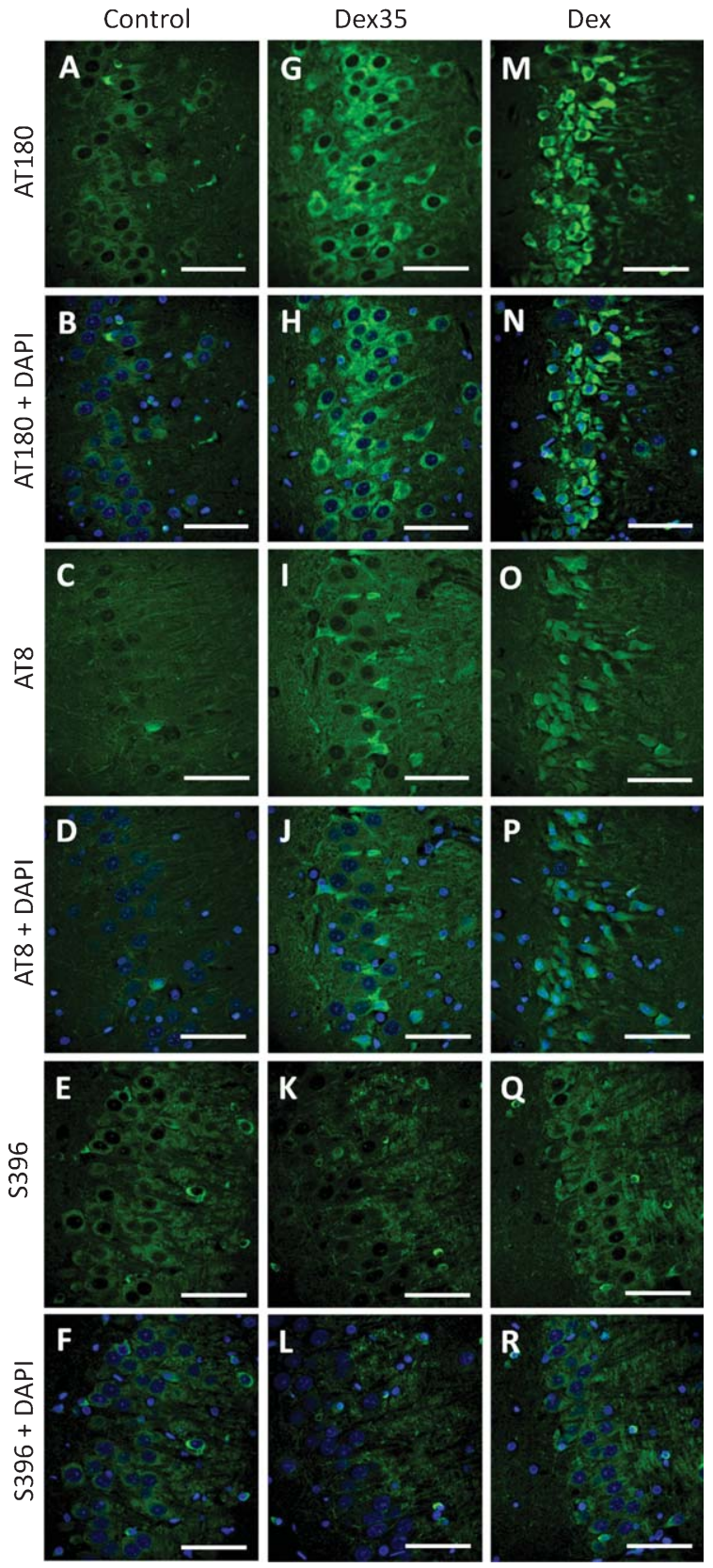

Fig. 2. Representative fluorescent photomicrographs of hippocampal sagittal sections with antibodies AT180, AT8, and S396. Fluorescence images of hippocampal sagittal sections are shown with AT180 (Green, $A, G, M$ ), AT8 (Green, $C, I, O$ ), S396 (Green, $E, K, Q)$, or merged with DAPI (Green-Blue, $B, H, N(A T 180) ; D, J, P$ (AT8); F,L,R (S396)) for the following conditions: Control (A-F); Dexmedetomidine with temperature maintenance (Dec35; G-L); Dexmedetomidine without temperature maintenance (Dex; M-R). All images were taken at 400x magnification.

The results indicate that dexmedetomidine may exert direct effects on the phosphorylation of tau, instead of only inducing tau phosphorylation via hypothermia. 
Dexmedetomidine induced tau phosphorylation in the rat hippocampus and cortex under hypothermic and normothermic conditions

As we found positive immunoreactivity for AT8 and AT180 in CA3 hippocampal neurons, we further confirmed the results by western blot analysis. Phosphorylation of tau protein, as detected by specific antibodies to the AT8, AT180, and S396 epitopes were compared by using the ratio of their levels to total tau protein (panTau) after exposure of dexmedetomidine for $2 \mathrm{~h}$. The pattern of tau phosphorylation in hippocampus from western blot was similar to that of immunofluorescent staining. The AT8, AT180, and
S396 immunoreactivity showed no significant changes in sham group when comparing with the control group in hippocampus. Dexmedetomidine-induced hypothermia significantly triggered phosphorylation of tau at Ser202/Thr205 (AT8) $(108.6 \pm 6.7 \%)$ and Thr231 (AT180) $(113.3 \pm 16.8 \%)$ when compared to the sham group (Fig. 3a). In the Dex35 group, tau phosphorylation at Ser202/Thr205 (AT8) (32.6 $\pm 12.3 \%)$ and Thr231 (AT180) $(14.6 \pm 32.7 \%)$ were also significantly increased. Interestingly, significant decrease in tau phosphorylation at $\mathrm{S} 396(50.8 \pm 4.3 \%)$ was found in Dex35 group compared to that of sham. These data are consistent with the results from immunofluorescent staining. The results implicate that phosphorylation at a

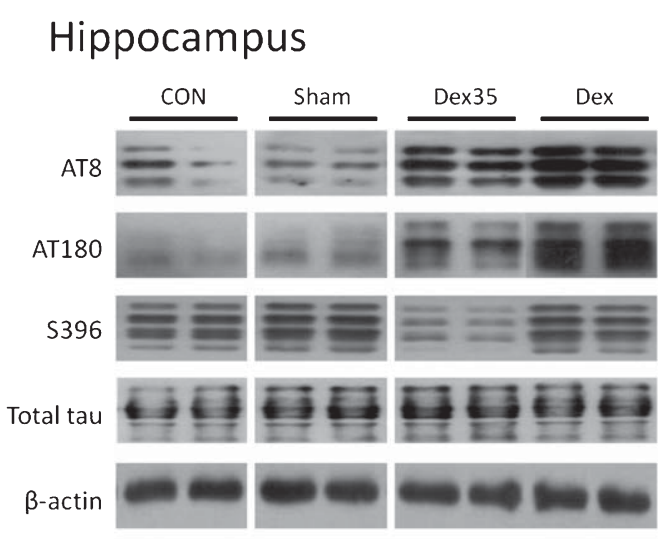

b

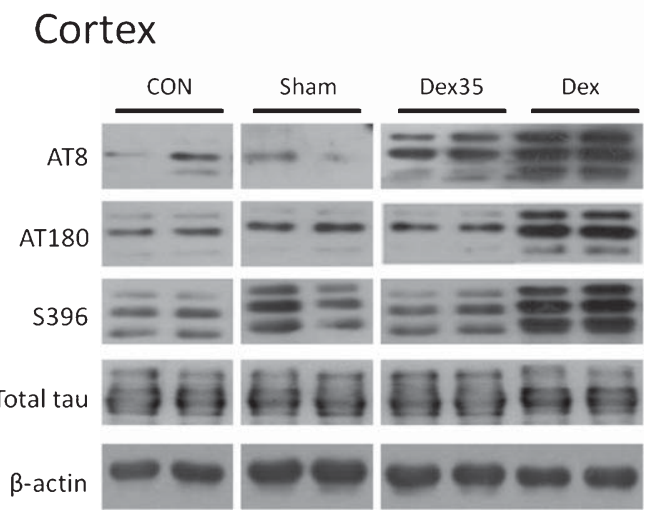

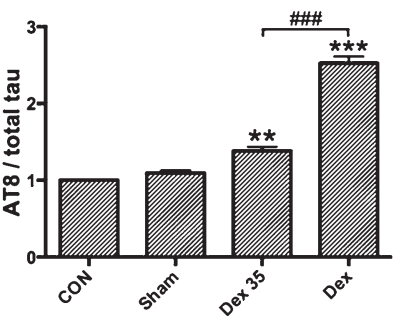
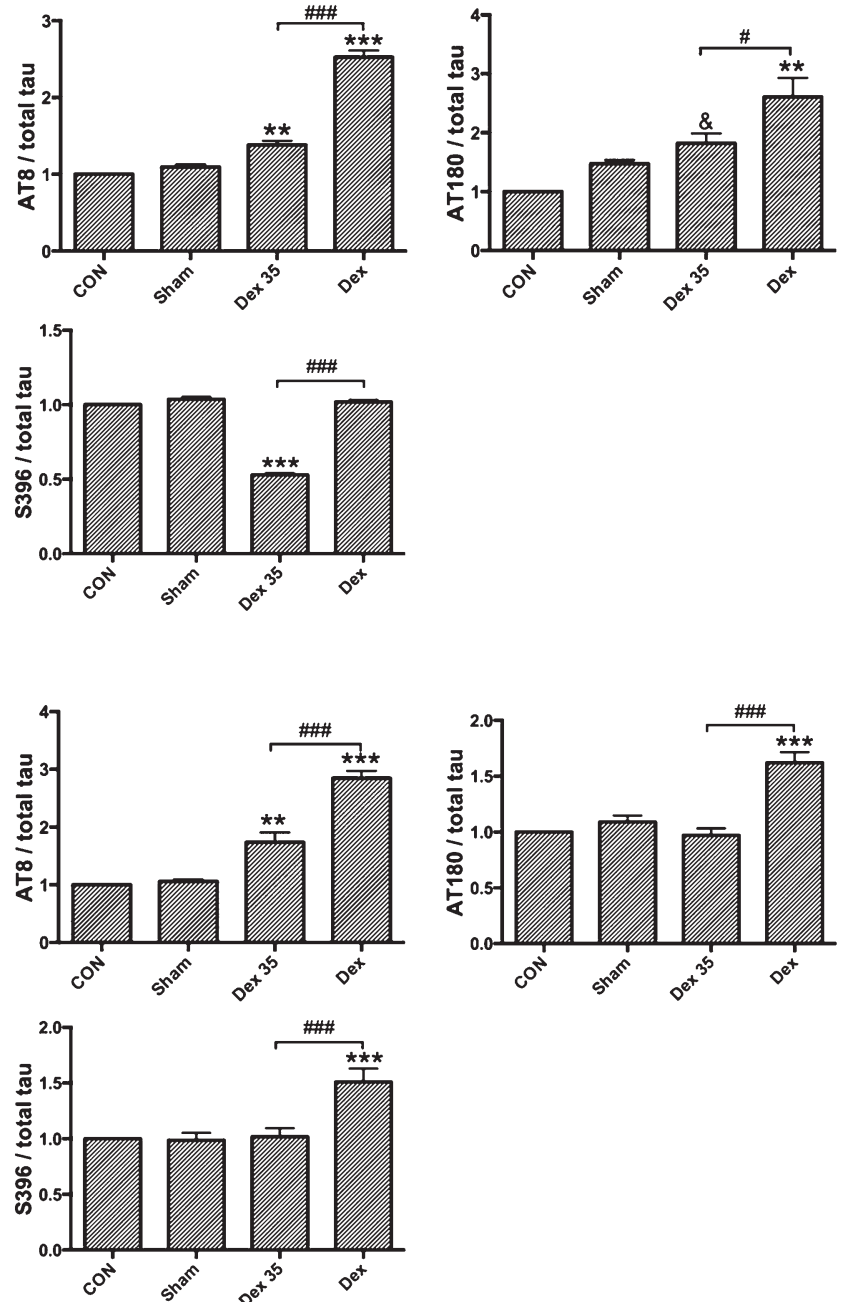

Fig. 3. Tau phosphorylation in a) rat hippocampus and b) cortex following $2 \mathrm{~h}$ of anesthesia and $1 \mathrm{~h}$ recovery. Results are presented as ratio of the control (CON). Tau phosphorylation were determined using antibodies directed at the AT8 (Ser202 + Thr205), AT180 (Thr231), and S396 sites. Data are expressed as mean $\pm \mathrm{SD},{ }^{*} p<0.05,{ }^{* *} p<0.01$ and ${ }^{* * *} p<0.001$ versus CON. ${ }^{*} p<0.05,{ }^{\& \&} p<0.01$, and \&\&\& $p<0.001$ versus Sham. CON, control; Sham, vehicle injection; Dex 35, Dexmedetomidine with temperature maintenance; Dex, Dexmedetomidine without temperature maintenance. 
S396 may occur after exposure to dexmedetomidine with hypothermia or dexmedetomidine with controlled temperature.

To determine whether there was any regional difference in tau phosphorylation, the levels of tau phosphorylation as detected by AT8, AT180, and S396 antibodies in the cortex as well as hippocampus were compared. In the cortex, no significant change in the immunoreactivity of AT8, AT180, and S397 was found in sham group comparing with control group. Dexmedetomidine-induced hypothermia significantly increased immunoreactivity at AT8 $(178.9 \pm 33.1 \%)$, AT180 (45.1 $\pm 16.8 \%)$, and S396 (52.2 $\pm 22.9 \%)$ epitopes, when compared with the sham group (Fig. 3b). Of note, a significant increase in immunoreactivity at AT8 $(68.0 \pm 13.3 \%)$, but not AT180 and S396 was observed in Dex35 group compared to the sham group. Nevertheless, the hypothermia-induced increase in tau phosphorylation at AT8 was greater than that in the Dex35 group. Again, these data demonstrated that tau phosphorylation at Ser202/Thr205 (AT8) following dexmedetomidine occurs in the cortex, under both normothermic and hypothermic conditions.

\section{Impact of dexmedetomidine on tau kinases and phosphatase in the hippocampus and cortex under hypothermic and normothermic conditions}

To further investigate how dexmedetomidine induced phosphorylation of tau in normothermic and hypothermic conditions, we analyzed different kinases including glycogen synthase kinase- $3 \beta$ (GSK3 $\beta$ ), protein kinase $\mathrm{B}(\mathrm{Akt})$, extracellular signal-regulated kinase (ERK), and c-Jun N-terminal kinase (JNK) and protein phosphatase $2 \mathrm{~A}(\mathrm{PP} 2 \mathrm{~A})$ in rats after exposure to dexmedetomidine for $2 \mathrm{~h}$ with or without maintenance of temperature.

It has been shown that GSK3 $\beta$ is responsible for phosphorylating tau [22]. The activity of GSK3 $\beta$ was attenuated by phosphorylation at Ser9 and enhanced by phosphorylation at Tyr216 [22]. In the hippocampus, significantly increase in the phosphorylation of GSK3 $\beta$ at Ser9 was found in both Dex group $(28.0 \pm 17.2 \%, p<0.05)$ and Dex35 group $(54.8 \pm 25.4 \%, p<0.0001)$ when compared to the sham group (Fig. 4a). In contrast, there was no difference in the phosphorylation of GSK3 $\beta$ at Tyr216 in both groups. In the cortex, similar results were observed, phosphorylation of GSK3 $\beta$ at Ser9 was increased significantly in Dex group $(70.5 \pm 38.1 \%, p<0.001)$ and Dex35 group $(116.7 \pm 37.4 \%, p<0.0001)$ (Fig. 4b). There was no significant change in the phosphorylation of GSK3 $\beta$ at Tyr216 of both anesthetic groups. This data indicates that induced hypothermia following exposure to dexmedetomidine may exert an inhibitory effect on the activity of GSK3 $\beta$ in both hippocampus and cortex. Most importantly, dexmedetomidine may also exert a direct inhibitory effect on the activity of GSK3 $\beta$ in both hippocampus and cortex.

Akt is a upstream kinase of GSK $3 \beta$ and regulates the activity of GSK3 $\beta$ via phosphorylating Ser9 site [22]. Moreover, it can enhance tau phosphorylation directly [23]. In the hippocampus, significant increase in Akt phosphorylation was found in the Dex group $(50.2 \pm 33.5 \%)$ only (Fig. $4 a)$. In the cortex, no significant change for Akt phosphorylation was seen in the Dex group (Fig. 4b). Interestingly, significant decrease in Akt phosphorylation in the Dex35 group $(17.2 \pm 7.3 \%)$ as compared to Dex was found. These data suggest that dexmedetomidine-induced hypothermia triggers Akt activity in the hippocampus, but not in the cortex, and may be associated with inhibition of GSK3 $\beta$ as shown before by increasing its phosphorylation at Ser9. Nevertheless, the differential responses of Akt phosphorylation levels in different brain regions after exposure to dexmedetomidine may also suggest that Akt is not the sole upstream regulator of GSK3 $\beta$.

ERK and JNK are two best-known MAPK pathways activated by mitogenic stimuli and cellular stress involved in AD [24]. Moreover, they also play important roles in phosphorylating tau in both physiological and pathological states $[25,26]$. Therefore, we examined the phosphorylation levels of these kinases after the exposure to dexmedetomidine. We observed a significant increase in phosphorylation of ERK in the Dex group $(26.5 \pm 16.2 \%, p<0.01)$ comparing with sham group in hippocampus. However, no significant change was found in the Dex35 group in both regions (Fig. 4a \& b). In contrast, phosphorylation of JNK was significantly increased in Dex in hippocampus and cortex. There was no significant different in the Dex35 group in both regions. These results demonstrated that dexmedetomidine might have different impacts on MAPK pathways in hypothermic and normothermic conditions.

PP2A is the key inhibitory phosphatase in the tau pathology [27] and tau hyperphosphorylation can be attributed to the inhibitory changes on Ser/Thr protein phosphatase (PP) activity induced by anesthesia-induced hypothermia [28]. The activity of PP2A is inactivated by phosphorylation at Y307 [29]. Therefore, the immunoreactivity of PP2A was examined whether it is contributed to the 
a

Hippocampus
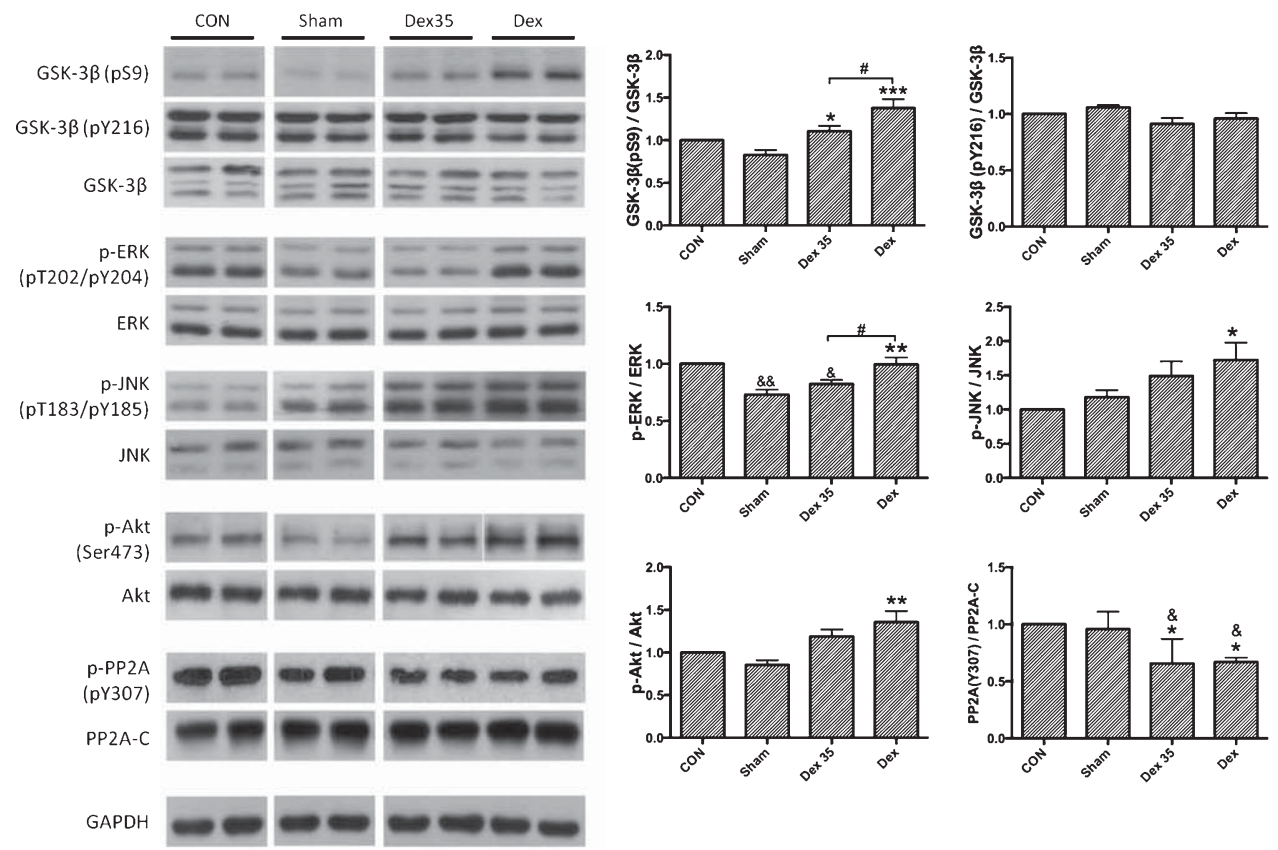

b
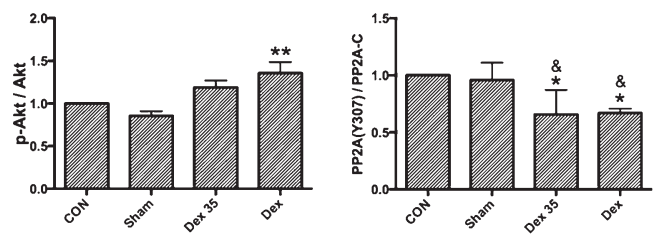

Cortex
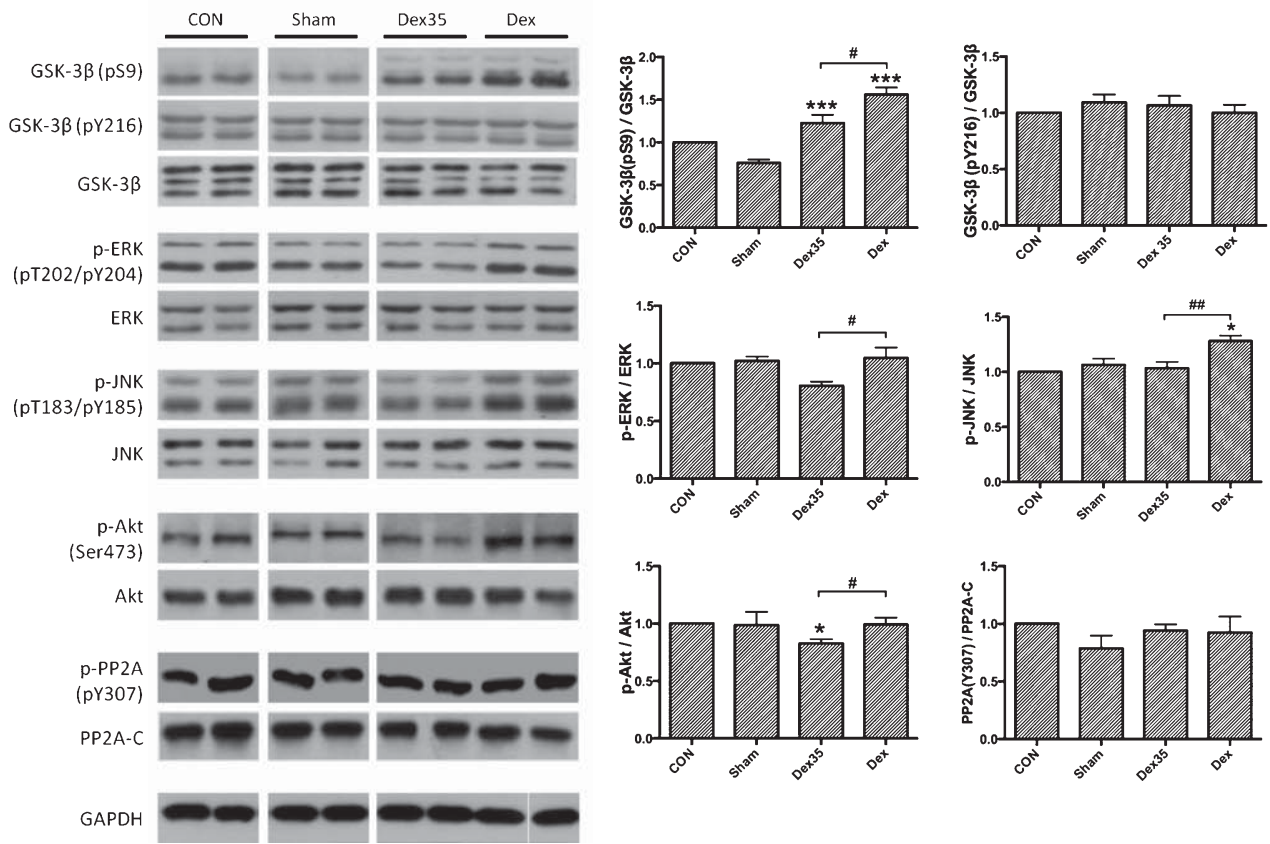

Fig. 4. Effect of dexmedetomidine on tau kinases in a) rat hippocampal and b) cortical tissues following $2 \mathrm{~h}$ of anesthesia and $1 \mathrm{~h}$ recovery. Results are presented as ratio of the control (CON). Tau phosphorylation were determined using antibodies directed at the phospho-GSK3 $\beta$ (S9), phospho-GSK3 $\beta$ (Y216), phospho-ERK1/2, total ERK, phospho-JNK, total JNK, phospho-AKT, total AKT, phospho-PP2A (Y307), PP2A-C, and GAPDH. Data are expressed as mean $\pm \mathrm{SD},{ }^{*} p<0.05,{ }^{* *} p<0.01$, and ${ }^{* * *} p<0.001$ versus $\mathrm{CON}$. ${ }^{\&} p<0.05$, \&\& $p<0.01$, and \&\&\& $p<0.001$ versus Sham. CON, control; Sham, vehicle injection; Dex 35, Dexmedetomidine with temperature maintenance; Dex, Dexmedetomidine without temperature maintenance. 
dexmedetomidine or hypothermia-induced hyperphosphorylation of tau. In the hippocampus, significant decrease in immunoreactivity at PP2A was found in both Dex $(71.5 \pm 14.1 \%, p<0.05)$ and Dex35 $(68.7 \pm 21.0 \%)$ (Fig.4a). However, in the cortex, no significant change in immunoreactivity at PP2A was observed in either Dex or Dex35 (Fig. 4b). These results reveal that tau hyperphosphorylation may be attributed to the inhibitory effect of dexmedetomidineinduced hypothermia on phosphatase activity. Most importantly, dexmedetomidine can also exert a direct inhibitory effect on PP2A activity in the hippocampus, which may contribute to tau hyperphosphorylation in the hippocampus.

In summary, these data suggest that dexmedetomidine-induced hypothermia leads to inhibition of GSK3 $\beta$ and activation of JNK in both the hippocampus and cortex. However, activation of Akt and ERK occurred in the hippocampus experiencing hypothermia. Moreover, dexmedetomidine may attenuate activation of Akt in the cortex. The results indicate that kinase activation is probably not the mechanism leading to tau hyperphosphorylation after exposure to dexmedetomidine. On the other hand, the results also demonstrate that dexmedetomidine led to a reduction in PP2A activity in the hippocampus under both hypothermic and normothermic conditions, which may contribute to tau hyperphosphorylation.

\section{DISCUSSION}

Dexmedetomidine exhibits a substantial anesthetic sparing effect clinically and can also independently induce sedation without respiratory depression and less cognitive dysfunction than GABA agonists [30]. It is, therefore, widely used for different types of surgery [31] and for sedation in the ICU. There are many studies examining the direct effect of "conventional" sedative/anesthetic agents on neurons in vitro and in vivo many of which are purported to act on GABA receptors. However, comparatively little data is available regarding the effects of this an alpha-2 receptor agonist on tau proteins. Preliminary results from Whittington and co-workers have shown that there is an increase in phosphorylation of tau at several sites under normothermic conditions in the hippocampus [32]. In the present study, we investigated the direct and indirect impacts of dexmedetomidine on tau phosphorylation by both in vitro and in vivo experiments. Among all the phosphorylation sites of tau, we focused on the phosphorylation site at AT8, AT180, and S396 as several other groups suggested the potential linkage of these epitopes with anesthesia induced effects $[8,32$, 33]. Despite dexmedetomidine having a very different mechanism of action compared with other anesthetics, our findings indicate that dexmedetomidine can increase tau phosphorylation in the hippocampus and cortex via hypothermia-dependent and -independent modes of action. In other words, hypothermia may not be the only explanation for tau phosphorylation induced by dexmedetomidine. These results support previous findings showing that different types of anesthetic agents, including propofol and sevoflurane, promote tau phosphorylation directly under normothermic conditions [6-8].

Our data indicate that $1 \mathrm{~h}$ of exposure of dexmedetomidine under normothermic conditions is sufficient to induce tau hyperphosphorylation in a primary culture of cortical neurons. However, the direct effect of dexmedetomidine on the phosphorylation of tau in differentiated cortical neurons is site-specific and transient. Dexmedetomidine-induced phosphorylation of tau occurs at S396 only, and such effect gradually returns back to the levels seen in controls after $6 \mathrm{~h}$ of exposure. Unless high doses $(20 \mu \mathrm{M})$ are used, a longterm effect of dexmedetomidine on phosphorylation of tau cannot be detected.

In quite a number of studies, induction of anesthesia was accomplished by administrating a large bolus without deliberately controlling the depth of anesthesia. This pattern of administering anesthetics in animals is different to that used in clinical practice [3, $6,8]$. Intraperitoneal injection of anesthetics is unreliable as injected contents may be inadvertently placed into the gut, abdominal fat, or other subcutaneous tissues. Furthermore, very deep anesthesia may require a certain time to ensure that drugs are absorbed in the body. The use of rats instead of mice in vivo models made it allow technically feasible to induce and maintain anesthesia intravenously. This enabled titration of the dose according to the response of the animal, a situation more closely mimicking the clinical situation. Using doses determined by our pilot study (data not shown), our delivery method can possibly avoid an initial period of excessively deep anesthesia associated with the bolus effect of intraperitoneal induction. Although not ideal, this enables some degrees of control of the dose of anesthetics over blood levels and, hence, depth of anesthesia.

Our results from in vivo studies demonstrate that the effects of dexmedetomidine on the phosphorylation of tau depend not only on the phosphorylation site but the regions of the brain being examined. 
Dexmedetomidine induced a robust hyperphosphorylation of tau, as demonstrated by immunohistochemical and western blot analysis. We found that tau phosphorylation recognized by AT8 and AT180 antibodies accumulated in CA3 hippocampal neurons under both hypothermic and normothermic conditions, demonstrating that hypothermia induced by anesthetics cannot fully explain how phosphorylation of tau occurs. Nevertheless, our results also demonstrate that hypothermia may exert a synergistic effect on tau phosphorylation at AT8 and AT180 sites triggered by dexmedetomidine. However, since the response of S396 phosphorylation was somewhat different to that of AT8 and AT180, phosphorylation at S396 may be an indicator of the effects of dexmedetomidine or hypothermia. It has been reported that not all tau phosphorylation events lead to a decrease in binding ability of tau to microtubules [34, 35]. Phosphorylation of tau at S396 and/or S404 does not significantly affect the affinity of tau for microtubules. Instead, phosphorylation at these sites may increase the propensity of tau to oligomerize and enhance the fibrillogenic properties of tau [34-36]. Of note, our findings here may implicate that the direct impact on tau phosphorylation at different phospho-epitopes by exposure to dexmedetomidine for $2 \mathrm{~h}$ could promote the dissociation of tau from microtubules but not increase the fribrillogenic properties of tau in the hippocampus. Further investigation is required to clarify the impact of dexmedetomidine on tau polymerization. Besides the hippocampus, hyperphosphorylation of tau was also demonstrated in the cortex after anesthesia with dexmedetomidine. Similar results were found in the tau phosphorylation at the AT8 and AT180 phospho-epitopes except that there was no hyperphosphorylation at the AT180 epitope under normothermia. This may suggest a different vulnerability between neurons from the two regions.

Phosphorylation of tau is regulated by several kinases and phosphatases. There are two main pathways involved in this process, including Akt/GSK3 kinases and mitogen-activated protein kinases (MAPK) pathways. Therefore, we examined the expression and/or activation of several enzymes in these pathways. Activation of Akt leads to inhibition of GSK3 $\beta$ activity by phosphorylation at Ser9 [22]. PP2A also contributed to the regulation of GSK3 $\beta$ activity by inactivate GSK3$\beta$ activity directly or indirectly through Akt dephosphorylation and activation [37]. Although the relationship between PP2A and GSK $3 \beta$ is not fully understood, PP2A and GSK3 $\beta$ affect activities of each other, which then impact tau phosphorylation directly and indirectly [29, 37, 38]. We demonstrated a reduction in inhibition of PP2A activity in both anesthesia-induced hypothermic and normothermic conditions in the hippocampal region. In addition, a decrease in GSK3 $\beta$ activity was found in the hippocampal and cortical regions in anesthesiainduced hypothermic and normothermic conditions. These results suggest that the reduction of PP2A activity and inhibition of GSK3 $\beta$ activity may be due not only to the induced hypothermia but also from the anesthetics itself. In AD brains, reduced phosphatase PP2A and increased in tau protein kinase GSK3 $\beta$ activity have been suggested to be involved in excessive tau phosphorylation [22, 29]. However, the opposite results found here indicate that the effects of dexmedetomidine on tau phosphorylation may not be primarily accomplished by altering the activity of PP2A and GSK3 $\beta$.

ERK and JNK have also been reported to be involved in tau phosphorylation. These two kinases are able to phosphorylate tau in a manner similar to paired helical filaments-like tau phosphorylation observed in AD brain [39]. Under normal physiological conditions, ERK plays a critical role in synaptic plasticity, learning, and memory in the hippocampus [39, 40]. Moreover, ERK has been reported to be involved in cytoskeletal reorganization as half of the ERKs are bound to the cytoskeleton in activated fibroblasts [40, 41]. On the other hand, the JNK pathway is usually activated by cellular stress such as oxidative stress [39]. In our study, the increased levels of p-ERK and the upregulation of JNK activity were observed in hypothermic conditions only, indicating the anesthetic alone may not directly trigger activation of ERK and JNK. Taken together, our results demonstrate that there is a dysregulation in MAPK, and any dysregulation of the MAPK pathways may lead to deleterious consequences in cognitive functions. For example, ERK participates in synaptic plasticity and learning and memory functions [39]. Imbalance in the MAPK pathway after anesthesia has been demonstrated in another investigation using sevoflurane instead [7]. This suggests that repeatedly exposure to dexmedetomidine may perturb MAPK, and hence cognitive function. This warrants further investigation.

In summary, in examining the direct and indirect impact of exposure to dexmedetomidine on tau phosphorylation using in vitro and in vivo models and the effects on the expression and activation of several tau kinases, we have found that the effect of this sedative agent on tau protein phosphorylation is site specific. Hypothermia cannot entirely explain why 
dexmedetomidine can trigger phosphorylation of tau. As our results show differential effects of dexmedetomidine on different brain regions, this may open a new avenue for studies focusing on different brain regions for their corresponding functions when investigating the effects of anesthetics.

\section{ACKNOWLEDGMENTS}

This project was partly supported by HKU Seed Funding Programme for Basic Research (201111159101) to GTCW, 201311159171 to RCCC, HKU Alzheimer's Disease Research Network under Strategic Research Theme on Ageing, and a generous donation from Ms. Kit Wan Chow.

Authors' disclosures available online (http://www.jalz.com/disclosures/view.php?id=2571).

\section{REFERENCES}

[1] Buée L, Bussière T, Buée-Scherrer V, Delacourte A, Hof PR (2000) Tau protein isoforms, phosphorylation and role in neurodegenerative disorders. Brain Res Rev 33, 95-130.

[2] Fodale V, Santamaria LB, Schifilliti D, Mandal PK (2010) Anaesthetics and postoperative cognitive dysfunction: A pathological mechanism mimicking Alzheimer's disease. Anaesthesia 65, 388-395.

[3] Run X, Liang Z, Zhang L, Iqbal K, Grundke-Iqbal I, Gong CX (2009) Anesthesia induces phosphorylation of tau. J Alzheimers Dis 16, 619-626.

[4] Dong Y, Wu X, Xu Z, Zhang Y, Xie Z (2012) Anesthetic isoflurane increases phosphorylated tau levels mediated by caspase activation and Abeta generation. PLoS One 7, e39386.

[5] Ikeda Y, Ishiguro K, Fujita SC (2007) Ether stress-induced Alzheimer-like tau phosphorylation in the normal mouse brain. FEBS Lett 581, 891-897.

[6] Planel E, Richter KE, Nolan CE, Finley JE, Liu L, Wen Y, Krishnamurthy P, Herman M, Wang L, Schachter JB, Nelson RB, Lau LF, Duff KE (2007) Anesthesia leads to tau hyperphosphorylation through inhibition of phosphatase activity by hypothermia. J Neurosci 27, 3090-3097.

[7] Le Freche H, Brouillette J, Fernandez-Gomez F-J, Patin P, Caillierez R, Zommer N, Sergeant N, Buée-Scherrer V, Lebuffe G, Blum D, Buée L (2012) Tau phosphorylation and sevoflurane anesthesia: An association to postoperative cognitive impairment. Anesthesiology 116, 779-787.

[8] Whittington RA, Virág L, Marcouiller F, Papon M-A, Khoury NBE, Julien C, Morin F, Emala CW, Planel E (2011) Propofol directly increases tau phosphorylation. PLoS One 6, e16648.

[9] Mantz J, Josserand J, Hamada S (2011) Dexmedetomidine: New insights. Eur J Anaesthesiol 28, 3-6.

[10] Carollo DS, Nossaman BD, Ramadhyani U (2008) Dexmedetomidine: A review of clinical applications. Curr Opin Anesthesiol 21, 457-461.

[11] Hoy S, Keating G (2011) Dexmedetomidine. Drugs 71, 1481-1501.

[12] Engelhard K, Werner C, Kaspar S, Möllenberg O, Blobner M, Bachl M, Kochs E (2002) Effect of the $\alpha 2$-agonist dexmedetomidine on cerebral neurotransmitter concentrations during cerebral ischemia in rats. Anesthesiology 96, 450-457.
[13] Huang R, Chen Y, Yu A, Hertz L (2000) Dexmedetomidineinduced stimulation of glutamine oxidation in astrocytes: A possible mechanism for its neuroprotective activity. J Cereb Blood Flow Metabo 20, 895-898.

[14] Engelhard K, Werner C, Eberspächer E, Bachl M, Blobner M, Hildt E, Hutzler P, Kochs E (2003) The effect of the $\alpha 2$-agonist dexmedetomidine and the $\mathrm{N}$-methyl-D-aspartate antagonist $\mathrm{S}$ (+)-ketamine on the expression of apoptosis-regulating proteins after incomplete cerebral ischemia and reperfusion in rats. Anesth Analg 96, 524-531.

[15] Sanders RD, Xu J, Shu Y, Januszewski A, Halder S, Fidalgo A, Sun P, Hossain M, Ma D, Maze M (2009) Dexmedetomidine attenuates isoflurane-induced neurocognitive impairment in neonatal rats. Anesthesiology 110, 1077-1085.

[16] Ho YS, Yang X, Lau JC, Hung CH, Wuwongse S, Zhang Q, Wang J, Baum L, So KF, Chang RC (2012) Endoplasmic reticulum stress induces tau pathology and forms a vicious cycle: Implication in Alzheimer's disease pathogenesis. J Alzheimers Dis 28, 839-854.

[17] Ho YS, Yu MS, Yang XF, So KF, Yuen WH, Chang RC (2010) Neuroprotective effects of polysaccharides from wolfberry, the fruits of Lycium barbarum, against homocysteine-induced toxicity in rat cortical neurons. $J$ Alzheimers Dis $\mathbf{1 9}$, 813-827.

[18] Cheung YT, Zhang NQ, Hung CH, Lai CS, Yu MS, So KF, Chang RC (2011) Temporal relationship of autophagy and apoptosis in neurons challenged by low molecular weight beta-amyloid peptide. J Cell Mol Med 15, 244-257.

[19] Ma D, Hossain M, Rajakumaraswamy N, Arshad M, Sanders RD, Franks NP, Maze M (2004) Dexmedetomidine produces its neuroprotective effect via the alpha $2 \mathrm{~A}$-adrenoceptor subtype. Eur J Pharmacol 502, 87-97.

[20] Tung A, Herrera S, Fornal CA, Jacobs BL (2008) The effect of prolonged anesthesia with isoflurane, propofol, dexmedetomidine, or ketamine on neural cell proliferation in the adult rat. Anesth Analg 106, 1772-1777.

[21] Ho YS, Yang X, Yeung SC, Chiu K, Lau CF, Tsang AW, Mak JC, Chang RC (2012) Cigarette smoking accelerated brain aging and induced pre-Alzheimer-like neuropathology in rats. PLoS One 7, e36752.

[22] Planel E, Sun X, Takashima A (2002) Role of GSK-3 $\beta$ in Alzheimer's disease pathology. Drug Dev Res 56, 491-510.

[23] Sadik G, Tanaka T, Kato K, Yamamori H, Nessa BN, Morihara T, Takeda M (2009) Phosphorylation of tau at Ser214 mediates its interaction with 14-3-3 protein: Implications for the mechanism of tau aggregation. J Neurochem 108, 33-43.

[24] Zhu X, Raina AK, Perry G, Smith MA (2004) Alzheimer's disease: The two-hit hypothesis. Lancet Neurol 3, 219-226.

[25] Nemoto T, Miyazaki S, Kanai T, Maruta T, Satoh S, Yoshikawa N, Yanagita T, Wada A (2010) Nav1.7$\mathrm{Ca} 2+$ influx-induced increased phosphorylations of extracellular signal-regulated kinase (ERK) and p38 attenuate tau phosphorylation via glycogen synthase kinase-3 $\beta$ : Priming of Nav1.7 gating by ERK and p38. Eur J Pharmacol 640, 20-28.

[26] Ploia C, Antoniou X, Sclip A, Grande V, Cardinetti D, Colombo A, Canu N, Benussi L, Ghidoni R, Forloni G, Borsello T (2011) JNK plays a key role in tau hyperphosphorylation in Alzheimer's disease models. J Alzheimers Dis 26, 315-329.

[27] Liu F, Grundke-Iqbal I, Iqbal K, Gong C-X (2005) Contributions of protein phosphatases PP1, PP2A, PP2B and PP5 to the regulation of tau phosphorylation. Eur J Neurosci 22, 1942-1950. 
[28] Planel E, Richter KEG, Nolan CE, Finley JE, Liu L, Wen Y, Krishnamurthy P, Herman M, Wang L, Schachter JB, Nelson RB, Lau L-F, Duff KE (2007) Anesthesia leads to tau hyperphosphorylation through inhibition of phosphatase activity by hypothermia. J Neurosci 27, 3090-3097.

[29] Martin L, Latypova X, Wilson CM, Magnaudeix A, Perrin M-L, Terro F (2013) Tau protein phosphatases in Alzheimer's disease: The leading role of PP2A. Ageing Res Rev 12, 39-49.

[30] Ebert TJ, Hall JE, Barney JA, Uhrich TD, Colinco MD (2000) The effects of increasing plasma concentrations of dexmedetomidine in humans. Anesthesiology 93, 382-394.

[31] Ramsay MA, Luterman DL (2004) Dexmedetomidine as a total intravenous anesthetic agent. Anesthesiology 101, 787 790.

[32] Whittington RA, Virág L, Wong K, Marcouiller F, Planel E (2011) Dexmedetomidine induces tau hyperphosphorylation in the mouse hippocampus under normothermic conditions. Annual Meeting of the American Society of Anesthesiologist, A685.

[33] Tan W, Cao X, Wang J, Lv H, Wu B, Ma H (2010) Tau hyperphosphorylation is associated with memory impairment after exposure to $1.5 \%$ isoflurane without temperature maintenance in rats. Eur J Anaesthesiol 27, 835-841.

[34] Stoothoff WH, Johnson GVW (2005) Tau phosphorylation: physiological and pathological consequences. Biochim Biophys Acta 1739, 280-297.
[35] Abraha A, Ghoshal N, Gamblin TC, Cryns V, Berry RW, Kuret J, Binder LI (2000) C-terminal inhibition of tau assembly in vitro and in Alzheimer's disease. J Cell Sci 113, 3737-3745.

[36] Cho J-H, Johnson GVW (2003) Glycogen synthase kinase $3 \beta$ phosphorylates tau at both primed and unprimed sites: Differential impact on microtubule binding. J Biol Chem 278, 187-193.

[37] Martin L, Magnaudeix A, Esclaire F, Yardin C, Terro F (2009) Inhibition of glycogen synthase kinase-3 $\beta$ downregulates total tau proteins in cultured neurons and its reversal by the blockade of protein phosphatase-2A. Brain Res $\mathbf{1 2 5 2}$, 66-75.

[38] Wang Y, Yang R, Gu J, Yin X, Jin N, Xie S, Wang Y, Chang H, Qian W, Shi J, Iqbal K, Gong C-X, Cheng C, Liu F (2014) Cross-talk between PI3K/AKT/GSK-3 $\beta$ and PP2A pathways determines tau hyperphosphorylation. Neurobiol Aging, doi: 10.1016/j.neurobiolaging.2014.07.035

[39] Zhu X, Lee HG, Raina AK, Perry G, Smith MA (2002) The role of mitogen-activated protein kinase pathways in Alzheimer's disease. Neurosignals 11, 270-281.

[40] Robinson MJ, Cobb MH (1997) Mitogen-activated protein kinase pathways. Curr Opin Cell Biol 9, 180-186.

[41] Reszka AA, Seger R, Diltz CD, Krebs EG, Fischer EH (1995) Association of mitogen-activated protein kinase with the microtubule cytoskeleton. Proc Natl Acad Sci U S A 92, 8881-8885. 